\title{
Simultaneous use of individual and joint regularization terms in compressive sensing: Joint reconstruction of multi-channel multi-contrast MRI acquisitions
}

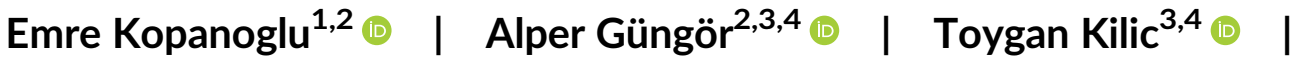

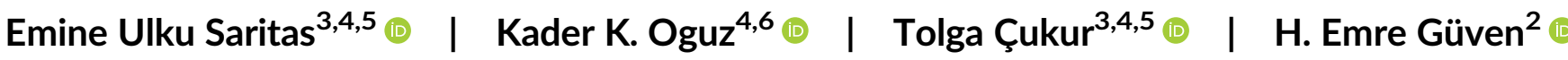

${ }^{1}$ Cardiff University, Brain Research Imaging Centre (CUBRIC), School of Psychology,

Cardiff University, Cardiff, UK

${ }^{2}$ ASELSAN Research Center, Ankara, Turkey

${ }^{3}$ Department of Electrical and Electronics Engineering, Bilkent University, Ankara, Turkey

${ }^{4}$ National Magnetic Resonance Research Center (UMRAM), Bilkent University, Ankara, Turkey

${ }^{5}$ Neuroscience Program, Sabuncu Brain Research Center, Bilkent University, Ankara, Turkey

${ }^{6}$ Department of Radiology, Hacettepe University, Ankara, Turkey

\section{Correspondence}

Emre Kopanoglu, CUBRIC, Cardiff University, Maindy Road. Cardiff, CF24 4HQ, UK.

Email: emre.kopanoglu@gmail.com

Funding information

The Science Academy, Turkey, Grant/Award Number: BAGEP Award; European Molecular Biology Organization Installation Grant, Grant/ Award Number: 3028; Turkish Scientific and Technological Research Council (TÜBITAK), Grant/Award Number: 3151068; Turkish Academy of Sciences (TUBA), Grant/Award Number: GEBIP 2015 Fellowship
Multi-contrast images are commonly acquired together to maximize complementary diagnostic information, albeit at the expense of longer scan times. A time-efficient strategy to acquire high-quality multi-contrast images is to accelerate individual sequences and then reconstruct undersampled data with joint regularization terms that leverage common information across contrasts. However, these terms can cause features that are unique to a subset of contrasts to leak into the other contrasts. Such leakage-of-features may appear as artificial tissues, thereby misleading diagnosis. The goal of this study is to develop a compressive sensing method for multichannel multi-contrast magnetic resonance imaging (MRI) that optimally utilizes shared information while preventing feature leakage. Joint regularization terms group sparsity and colour total variation are used to exploit common features across images while individual sparsity and total variation are also used to prevent leakage of distinct features across contrasts. The multi-channel multi-contrast reconstruction problem is solved via a fast algorithm based on Alternating Direction Method of Multipliers. The proposed method is compared against using only individual and only joint regularization terms in reconstruction. Comparisons were performed on singlechannel simulated and multi-channel in-vivo datasets in terms of reconstruction quality and neuroradiologist reader scores. The proposed method demonstrates rapid convergence and improved image quality for both simulated and in-vivo datasets. Furthermore, while reconstructions that solely use joint regularization terms are prone to leakage-of-features, the proposed method reliably avoids leakage via simultaneous use of joint and individual terms, thereby holding great promise for clinical use.

LIST OF ABBREVIATIONS: MRI, magnetic resonance imaging; PI, parallel imaging; CS, compressive sensing; SAR, specific absorption rate; TV, Total Variation; SIMIT, SIMultaneous use of Individual and joinT regularization terms for joint CS-PI reconstruction; ADMM, Alternating Direction Method of Multipliers; Indiv-only, Reconstruction method that uses only individual $\ell_{1}$ sparsity and TV terms; Joint-only, Reconstruction method that uses only joint terms CTV and group $\ell_{1}$-sparsity; ESPIRiT, Eigenvalue-based implementation of Iterative self-consistent parallel imaging reconstruction from arbitrary k-space; TVCMRI, Total Variation $\ell_{1}$ Compressed MR Imaging; RecPF, reconstruction from partial Fourier data (RecPF); GSMRI, Group-Sparse MRI; FCSA Fast Composite Splitting Algorithm; FCSA-MT, Multi Contrast FCSA; CTV, colour TV; gL1, Group $\ell_{1}$-sparsity, implemented as an $\ell_{2,1}$-norm; iTV, Individual TV; iL1, Individual $\ell_{1}$-sparsity; pSNR, peak signal-to-noise ratio; PD / T1 / T2, Proton density / T1- / T2- weighted; 1D / 2D, one- / two- dimensional; TE / TI / TR, echo / inversion / repetition time; FLAIR, fluid-attenuated inversion recovery; STIR, short-time inversion recovery; FOV, field-of-view; SSIM, structural similarity; R, Acceleration rate; ROI, Region-of-interest. 


\section{KEYWORDS}

compressive sensing, joint reconstruction, leakage-of-features, magnetic resonance imaging, multi contrast, parallel imaging, image reconstruction, joint regularization

\section{INTRODUCTION}

Multiple images of the same anatomy under the influence of different contrasts are often acquired in magnetic resonance imaging (MRI) to accumulate diagnostic information. Common examples include multi-contrast imaging with T1, T2, or PD weighting, parametric mapping, and diffusion weighted imaging. However, with each acquisition lasting several minutes, MRI exams can become impractically long and costly. Prolonged scan times also increase susceptibility to patient motion and necessitate cumbersome motion correction or image registration procedures. Therefore, scan-time reduction techniques are direly needed to limit cost, patient discomfort, and motion with increasing number of acquisitions.

Accelerated imaging approaches including parallel imaging (PI), ${ }^{1-6}$ multi-slice imaging ${ }^{7-9}$ localizing the excitation volume, ${ }^{10-17}$ dephasing outer volumes, ${ }^{18,19}$ localizing encoding to a sub-volume ${ }^{20-22}$ and compressive sensing $(\mathrm{CS})^{23-45}$ are promising solutions. Among these, CS has gained prominence in the last decade, as it does not require complicated excitation pulses with increased specific absorption rate (SAR) or additional hardware. Furthermore, CS is compatible with alternative approaches such as parallel imaging ${ }^{32,41}$ and simultaneous multi-slice imaging. ${ }^{46}$

Conventional CS techniques process each acquisition in a multi-contrast protocol individually. ${ }^{25-28}$ Yet, although tissues may appear at different signal levels in separate contrasts, the underlying tissue structure is shared among multi-contrast acquisitions. As such, multi-contrast images share common tissue boundaries, and they are likely compressible in similar transform domains. These observations have motivated researchers to investigate the benefits of jointly reconstructing multiple images of the same anatomy. ${ }^{29-40}$ Proposed application domains include dynamic MRI reconstructions that handle single-contrast acquisitions across time, ${ }^{35,36}$ multi-coil MRI reconstructions, ${ }^{32}$ multi-echo MRI reconstructions that handle repeated acquisitions with minor changes in contrast, ${ }^{33,37}$ fat-water separation ${ }^{38}$, multi-contrast MRI reconstructions that process multiple distinct contrasts, ${ }^{29-31}$ and even multi-modality reconstructions. ${ }^{34}$

Joint reconstructions aim to utilize the information shared across contrasts to improve image quality. Sparse recovery during joint reconstructions has been attempted with a multitude of regularization terms in literature. A group of studies have focused on aggregation of individual regularization terms on each separate image such as the well-known $\ell_{1}$-sparsity ${ }^{47}$ and Total Variation (TV) ${ }^{48}$ terms. In Ref.32, sparsity was promoted simultaneously across multiple receive channels by imposing $\ell_{1}$-sparsity on concatenated multi-channel dynamic MRI data. Ref.38 jointly reconstructed water and fat images from a multi-echo acquisition by minimizing the sum of individual regularization functions on each image. Ref.49 performed a quasi-joint reconstruction by spatially weighing the individual $e_{1}$-sparsity and Total Variation of an image using structural information extracted from a prior individually reconstructed image. The performance improvement with joint reconstruction depends on how shared information is leveraged against the information unique to each contrast. Classical individual regularization terms help preserve unique information in each contrast without leakage of distinct features across images, but reconstructions can be sub-optimally sensitive to shared information across contrasts. Meanwhile, joint regularization terms such as group sparsity ${ }^{50}$ and Colour TV ${ }^{51}$ that enforce $e_{1}$-sparsity and total variation on multiple images simultaneously have provided useful in several applications including parametric mapping, ${ }^{40}$ diffusion tensor imaging, ${ }^{39}$ multi-echo T2-weighted imaging ${ }^{29,33}$ and multi-contrast imaging. ${ }^{30,31,52,53}$ Ref.35 minimized the nuclear norm to exploit the temporal correlations whereas Ref.36 used Frobenius and nuclear norms in a blind compressed sensing approach to dynamic MRI. Variations of TV regularization such as total generalized variation, ${ }^{34}$ parallel sets ${ }^{54}$ and weighted joint (colour) TV ${ }^{55,56}$ have also been used for jointly reconstructing MR and PET images ${ }^{34,54,56}$ or for multi-contrast MRI. ${ }^{57,58}$ Joint regularization terms boost sensitivity for features that are common across acquisitions, but as a result they can reduce sensitivity for features that are unique to each acquisition, and a feature that is only prominent in a single acquisition may leak into reconstructions of other acquisitions. Appearance of such artificial features can severely impair diagnostic evaluations; therefore, multiacquisition reconstructions should be carefully investigated for leakage-of-features.

In this study, we propose a reconstruction method for multi-acquisition MRI, named SIMultaneous use of Individual and joinT regularization terms for joint CS-PI reconstruction (SIMIT). SIMIT leverages both joint and individual regularization terms to maximize sensitivity for shared features among contrasts as well as unique features of each contrast while preventing undesirable leakage-of-features. Specifically, colour TV $(\mathrm{CTV})^{51}$ and group $\ell_{1}$-sparsity ${ }^{50}$ are used to exploit common information across contrasts, and individual $\mathrm{TV}^{48}$ and $\ell_{1}$-sparsity ${ }^{47}$ are used to prevent leakage-of-features. SIMIT is demonstrated for multi-contrast imaging, where the resulting optimization problem is solved efficiently via an adaptation $^{59}$ of Alternating Direction Method of Multipliers (ADMM). ${ }^{27,60,61}$ First, SIMIT is compared against alternative reconstructions that only use individual $\ell_{1}$-sparsity and TV terms (Indiv-only) ${ }^{59}$ or only use the joint terms CTV and group $\ell_{1}$-sparsity (Joint-only), ${ }^{62}$ on a numerical phantom dataset. The phantom only included a single-channel receiver coil to isolate potential leakage artefacts. SIMIT is then compared against Indiv-only and Joint-only as well as ESPIRiT reconstructions ${ }^{63}$ on multi-channel in vivo datasets. The main contributions of this study are as follows: 1 ) We introduce the simultaneous use of individual and joint versions of regularization terms in a multi-channel multi-acquisition reconstruction problem. 2) We demonstrate improved image quality and reliability against leakage-of-features in accelerated multi-contrast MRI. Single-channel and multi- 
channel implementations of this method were presented in part in the 2017 and 2019 Annual Meetings of the International Society for Magnetic Resonance in Medicine. ${ }^{64,65}$ In Ref.64, a single-channel version of the method was presented on a single subject. In Ref.65, preliminary comparisons were performed between an earlier multi-channel implementation and ESPIRiT. Here, further to Refs.64, 65, we provide a detailed theoretical description of the multi-channel, multi-contrast reconstruction method, thoroughly investigate the benefits of simultaneously using individual and joint regularization terms as opposed to using only joint or only individual terms, demonstrate reconstruction performance across a broad range of acceleration rates and numbers of jointly reconstructed contrasts, and perform qualitative and quantitative comparisons against three state-of-the-art methods for in-vivo data acquired from $N=11$ participants.

\section{2 | METHODS}

\section{1 | Theory}

We propose to jointly reconstruct multi-contrast datasets by leveraging common information across contrasts via CTV and group sparsity $\left(\ell_{2,1^{-}}\right.$ norm, denoted by $g L 1$ ) regularization while preventing unwanted leakage artefacts via individual TV (iTV) and sparsity ( $\ell_{1}$-norm, denoted by iL1) regularization. The resulting optimization problem is:

$$
\begin{gathered}
\min _{x}\left[\alpha_{C T V} \operatorname{CTV}(|\boldsymbol{x}|)+\beta_{g L 1}\|\boldsymbol{x}\|_{2,1}+\gamma_{i T V} \sum_{i}^{k} T V\left(\left|\boldsymbol{x}^{(i)}\right|\right)+\theta_{i L 1} \sum_{i}^{k}\left\|\boldsymbol{x}^{(i)}\right\|_{1}\right] \\
\text { subject to }\left\|\boldsymbol{A}^{(i, j)} \boldsymbol{x}^{(i)}-\boldsymbol{y}^{(i, j)}\right\|_{2} \leq \epsilon_{i, j}, \quad i \in 1, \ldots, k ; j \in 1, \ldots, N_{c},
\end{gathered}
$$

where $k$ is the number of contrasts, $N_{c}$ is the number of channels (coils), $A^{(i, j)}, x^{(i)}, y^{(i, j)}$ and $\epsilon_{i, j}$ denote the encoding matrix, the reconstructed image vector, the received signal acquired through channel $j$ for contrast $i$ and the upper-bound for data-fidelity. Equation (2) denotes the data fidelity constraint for the $i^{\text {th }}$-contrast and $j^{\text {th }}$-channel. We prefer including data-fidelity as a constraint as opposed to a Lagrangian form, since $\epsilon_{i, j}$ can simply be set according to the noise level calculated from noise-only data (i.e., data acquired without RF excitation). The CTV, $\ell_{2,1}, \mathrm{TV}$, and $\ell_{1}$ regularization terms can be expressed as:

$$
\begin{aligned}
& \operatorname{CTV}(|\boldsymbol{x}|)=\sum_{n} \sqrt{\sum_{i=1}^{k}\left(\left(\nabla_{1}\left|x^{(i)}[n]\right|\right)^{2}+\left(\nabla_{2}\left|x^{(i)}[n]\right|\right)^{2}\right)}, \\
& \|\boldsymbol{x}\|_{2,1}=\sum_{n} \sqrt{\sum_{i=1}^{k}\left|x^{(i)}[n]\right|^{2}} \\
& \operatorname{TV}\left(\left|\boldsymbol{x}^{(i)}\right|\right)=\sum_{n} \sqrt{\left(\nabla_{1}\left|\boldsymbol{x}^{(i)}[n]\right|\right)^{2}+\left(\nabla_{2}\left|\boldsymbol{x}^{(i)}[n]\right|\right)^{2}} \\
& \left\|\boldsymbol{x}^{(i)}\right\|_{1}=\sum_{n}\left|\boldsymbol{x}^{(i)}[n]\right|
\end{aligned}
$$

where $\alpha_{C T V}, \beta_{g L 1}, \gamma_{i T V}, \theta_{i L 1}$ denote the respective regularization parameters. In (3), $\nabla_{1}, \nabla_{2}$ denote the image gradients in two orthogonal dimensions. Note that, all functions in (3) can trivially be extended to a higher number of dimensions as would be required for three-dimensional or dynamic acquisitions. The joint regularization terms $\ell_{2,1}$ and CTV combine the contrasts or their spatial derivatives, respectively, before regularizing the images. This allows an image with an inconspicuous tissue boundary that would normally be suppressed via TV regularization to retain this boundary, if the boundary is more prominent in another contrast. However, a prominent but unique feature in one contrast may lead to imperfect noise suppression in other contrasts, leading to misleading artificial tissues. I.e., joint regularization terms enhance reconstructions based on common properties across contrasts but may also lead to leakage of unique features across contrasts. Meanwhile, the individual regularization terms TV and $e_{1}$ suppress interference and noise based on the unique structural properties of each contrast, promising sparse recovery at higher undersampling rates without introducing unwanted feature transfer across contrasts. Therefore, the simultaneous use of the individual terms with the joint terms suppresses leakage-of-features due to the latter. While the individual and group sparsity terms can be applied in a transform domain that sparsifies the image, they are applied in the image domain in SIMIT, since empirical results in the development stages showed $>3 \mathrm{~dB}$ better peak signal-to-noise ratio (pSNR) when these terms were applied in the image domain rather than in Wavelet or Discrete Cosine Transform domains. For typical Wavelet transforms in MRI reconstruction, the $e_{1}$-term in Wavelet domain captures partly similar information to the TV term in image domain. This redundancy is further increased due to simultaneous use of individual and joint versions of regularization terms. In contrast, an $\ell_{1}$-term in the image domain can improve capture of unique prior information over the TV term. In this study, pSNR was calculated as the ratio of the maximum image intensity across voxels to the root-mean-squared error between the original $\left(\boldsymbol{x}_{0}\right)$ and the reconstructed $(\boldsymbol{x})$ images, and expressed in decibels: $\mathrm{pSNR}=20 \log _{10} \frac{\max (|\mathbf{x}|)}{\operatorname{RMS}\left(\left|\mathbf{x}_{0}-\mathbf{x}\right|\right)}$.

In the Supporting Information, we give the general ADMM formulation, show how the proposed optimization problem for multi-contrast MRI can be cast in this general formulation, and derive the update rules for implementation. The only parameter not shown in the body of the 
manuscript, $1 / \mu$, is the step-size parameter which determines the rate of convergence; a smaller $\mu$ means larger steps and faster convergence. ADMM is known to converge under mild conditions ${ }^{66}$ and the step size should be carefully selected to ensure good convergence behaviour, as the algorithm may diverge for very small $\mu$. An automated way of selecting this parameter is given in Ref. ${ }^{67}$ For non-convex problems, if the exact solution of each sub-problem is known, then the algorithm converges to a local minimum.

\section{2 | Undersampling masks and noise}

k-Space data were retrospectively undersampled in one (1D-acceleration) or two (2D-acceleration) phase-encode directions to demonstrate performance for two- and three-dimensional imaging, respectively (Figure 1). The central one-eighth section of k-space was fully-sampled. For 2Dacceleration, the diameter of the fully-sampled disc was set to one-eighth of the width of the k-space. Sampling masks were generated using probability distribution functions that decayed with a polynomial order of (R-2) or 3 (whichever is larger), where $R$ is the undersampling factor. ${ }^{25}$ The undersampling masks were identical across reconstruction methods but were different across contrasts.

The data-fidelity upper-bounds for SIMIT $\left(\epsilon_{i, j}\right)$ and its alternative variants (individual terms: Indiv-only; joint terms: Joint-only) were empirically set to half of the square root of the noise power in experimental reconstructions. Simulations were designed to investigate different factors that may affect performance. To isolate the effect of such factors on reconstruction performance, noiseless images were used for the simulations, unless specified otherwise.

\section{3 | Numerical phantom}

The numerical dataset was generated using a realistic individual-subject brain phantom that contained segmentation masks for eleven types of tissues. ${ }^{68}$ The original data for the phantom were acquired using a $1.5 \mathrm{~T}$ scanner with the following parameters: T1-weighted images (3D spoiled gradient echo): TR, $22 \mathrm{~ms}$; TE, $9.2 \mathrm{~ms}$; flip-angle, 30; resolution, $1 \mathrm{~mm}$ isotropic; PD- and T2-weighted images (turbo spin echo): TR, $3300 \mathrm{~ms}$; TE (PD/T2), 15/104 ms; resolution, $1 \mathrm{~mm}$ isotropic; number of slices, 62; slice thickness, $2 \mathrm{~mm}$; interslice gap, $0 \mathrm{~mm}$.

The following contrasts were simulated: PD-weighted (TE/TR: 17/2775 ms), T1-weighted (TE/TR: 14/575 ms), T2-weighted (TE/TR: 102/2775 ms), T1-weighted fluid-attenuated inversion recovery (FLAIR, TE/TI/TR: 17/1050/2775 ms), and T2-weighted short-time inversion recovery (STIR, TE/TI/TR: 17/240/2775 ms). Sinusoidal phase variations in the Anterior-Posterior direction were simulated to introduce image phase, and variations at different spatial frequencies were assumed for separate contrasts (Figure 2).

1D-undersampling

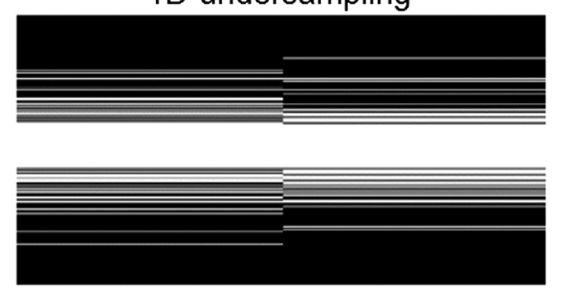

2D-undersampling

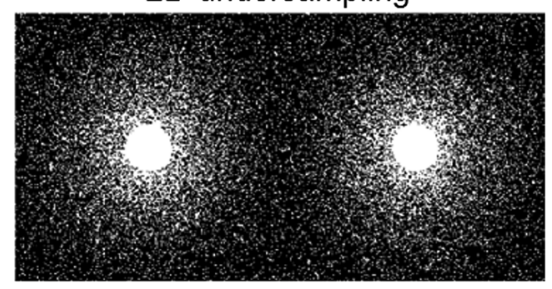

FIGURE 1 Two examples of 1D and 2Dundersampling masks

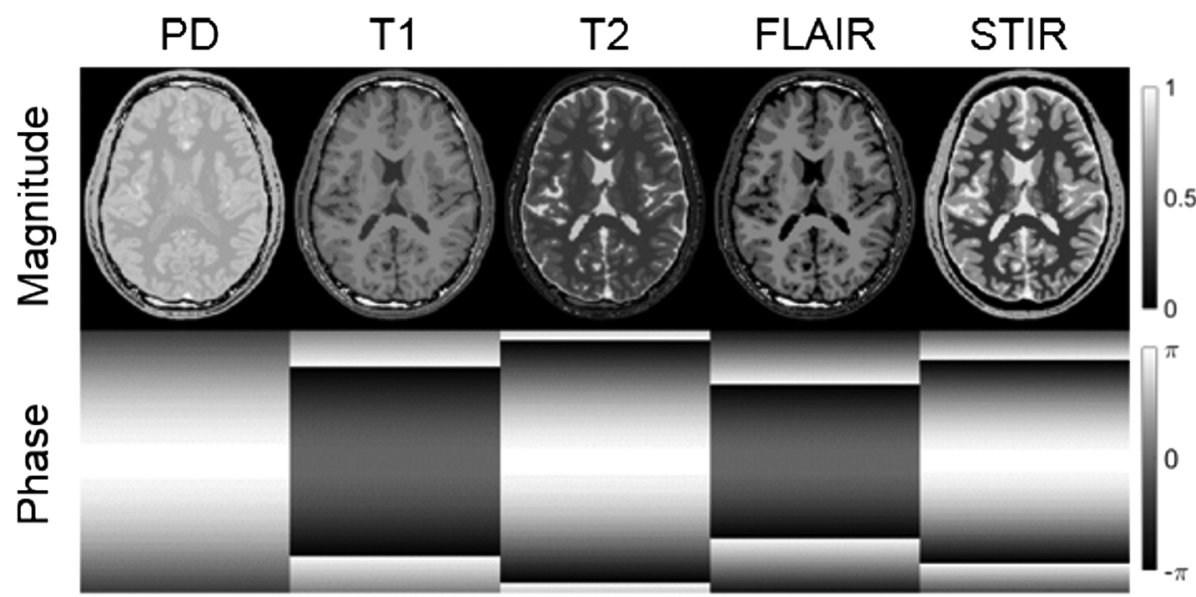

FIGURE 2 Magnitude (top) and phase (bottom) images from the numerical dataset for five different tissue contrasts: Proton-density (PD)-weighted, T1-weighted, T2-weighted, fluidattenuated inversion recovery (FLAIR) and short-TI inversion recovery (STIR) images 


\section{4 | In-vivo data}

Comparisons were made using in-vivo raw-data, acquired from $N=11$ participants using a $3 \mathrm{~T}$ scanner (Siemens Healthcare, Erlangen, Germany) with a 32-channel receiver-array head coil. Experimental procedures were approved by the local ethics committee and written informed consent was obtained from the participants. A field-of-view (FOV) of $192 \mathrm{~mm} \times 256 \mathrm{~mm} \times 176 \mathrm{~mm}$ (phase $\mathrm{x}$ readout $\mathrm{x}$ slice) and resolution of $1 \mathrm{~mm} \times$ $1 \mathrm{~mm} \times 2 \mathrm{~mm}$ were prescribed for all acquisitions. For T1-weighted acquisitions, an MP-RAGE sequence was used with $\mathrm{TE} / \mathrm{Tl} / \mathrm{TR}=3.87 / 1100 / 2000 \mathrm{~ms}$; flip-angle $=20^{\circ}$. For PD- and T2-weighted acquisitions, a turbo spin echo sequence was used with $\mathrm{TE}$ $\mathrm{TE}_{\mathrm{T} 2}=118 \mathrm{~ms}, \mathrm{TR}=1000 \mathrm{~ms}$, flip-angle=90 ; turbo-factor $=16$, echoes $/$ slice $=12$. Coil sensitivities were estimated using the approach in Ref. ${ }^{63}$

\section{5 | Parameter optimization and image normalization}

In CS reconstructions, resultant image quality depends on proper selection of regularization parameters (e.g., $\alpha_{C T V}, \beta_{g L 1}, \gamma_{i T V}, \theta_{i L 1}$, in SIMIT). These parameters are often optimized on held-out training data since fully-sampled data are not available in test subjects. Thus, parameter selection is expected to be suboptimal to varying degrees in practice. To allow for a controlled level of suboptimality, here we intentionally optimized parameters on a five-contrast numerical dataset but tested on datasets with fewer contrasts without dataset-specific optimization.

A mismatch between the image intensities in the training and test datasets may affect reconstruction quality. Here, raw k-space data for each acquisition were normalized such that the images reconstructed using Inverse Fourier Transform (simulations) or ESPIRiT (in-vivo reconstructions) for $R=1$ spanned the same intensity range, $[0,255]$. To optimize reconstruction performance, the regularization parameters for SIMIT, Indiv-only and Joint-only were separately optimized to maximize SSIM (structural similarity), which has been suggested to correlate highly with perceptual quality of visual images. ${ }^{69}$ Reconstructions were performed with each method for 500 iterations. Each contrast in the five-contrast numerical dataset was 1D-undersampled at $R=3$. An interval search algorithm was used with grid size: $11 \times 11$, depth: 3 , parameter range: 0.001 to 2.5 . The parameters in ${ }^{31}$ were used as initial values and the range was automatically expanded by the algorithm as necessary. The optimized parameters were 0.02 ( $e_{1}$-sparsity) and 1.14 (TV) for Indiv-only and 0.085 (Group $e_{1}$-sparsity) and 0.23 (CTV) for Joint-only.

Because the search space for SIMIT with four regularization terms is four-dimensional, the parameters optimized for Joint-only were used for the joint terms and the individual regularization parameters were manually tuned using the joint regularization parameters as the initial values. Note that, the joint regularization terms scale with $\sqrt{k}$ while the sum of the individual terms scales linearly with $k$. Because the parameters were optimized for a five-contrast dataset, parameters were scaled with $\sqrt{5 / k}$ and $5 / k$. Scaling with $\sqrt{1 / k}$ and $1 / k$ maintains balance among the regularization terms for an arbitrary number of contrasts $k$, while normalizing the scaling coefficients with $\sqrt{5}$ and 5 keep the coefficients unaltered for $k=5$, respectively. The optimization yielded $\alpha_{C T V}=0.19 / \sqrt{k}, \gamma_{i T V}=0.11 / k, \beta_{g L 1}=0.51 / \sqrt{k}, \theta_{i L 1}=9.13 / k$. The convergence speed parameter was empirically selected as $1 / \mu=\sqrt{N} / 10$, where $N$ is the number of image pixels.

For in vivo experiments, the original pixel intensities in T1-weighted images were approximately an order of magnitude smaller than those in PD- or T2-weighted images. Thus, the normalization of the data scaled the signal in T1-weighted images upwards, preventing a potential mismatch between the image intensities and the regularization parameters. However, because SNR remains constant after normalization, T1-weighted images had higher noise level compared to that of PD- and T2-images during joint reconstructions, leading to noisy reconstructions. To alleviate this issue, parameters for individual regularization terms $\left(e_{1}\right.$-sparsity and TV) were increased 5-fold for Indiv-only and SIMIT.

An adaptation of ESPIRiT with $\ell_{1}$-sparsity and TV terms was used to keep regularization terms as consistent as possible across methods under comparison. The parameters were optimized using the same approach as above, but because ESPIRiT uses a fundamentally different algorithm, the initial parameter range was adapted as 0.00005-0.125. We observed that optimized parameters yielded over-smoothing in in-vivo reconstruction, so the parameters were manually fine-tuned to increase visual acuity and pSNR, yielding $0.00025\left(\ell_{1}\right.$-sparsity) and 0.000625 (TV). A kernel size of $6 \times 6$ was used for ESPIRiT.

\subsection{Simulated data}

SIMIT was compared to Indiv-only and Joint-only. All methods used optimized parameters and 250 iterations. To assess reconstruction performance as a function of acceleration rate, methods were compared in terms of pSNR and SSIM for acceleration rates between $R=2$ and $R=5$ for 1D-acceleration and between $R=4$ and $R=15$ for $2 \mathrm{D}$-acceleration.

To investigate performance as a function of the number of acquisitions that are jointly reconstructed, SIMIT was performed on 2Daccelerated data with undersampling factors between $R=4$ and $R=15$. The number of contrasts was varied from 1 to 5 , and for each number all possible subsets of the five-contrast dataset were considered. pSNR and SSIM were averaged across 10 initializations of the undersampling mask for each case. pSNR and SSIM were also averaged across all subsets that contained a given contrast, e.g., the SSIM of the PD image was averaged across PD-T1, PD-T2, PD-FLAIR and PD-STIR for two-contrast joint reconstruction. 
Methods were also compared in terms of reconstruction time and stability of performance across undersampling masks and noise instances via a Monte-Carlo simulation with 250 runs. The five-contrast dataset was used with $1 D$-undersampling and $R=3$. Each run was performed with independent instances of noise and undersampling masks. Runtimes (excluding data-preparation) at each iteration were measured with the cputime command in Matlab (which excludes any parallel computing capabilities) and averaged across runs. SSIM and pSNR averaged across contrasts and runs were plotted as a function of cumulative runtime for each method. Bivariate Gaussian noise was added with a standard deviation equal to $10 \%$ of the mean intensity of k-space data across all contrasts. A second set of Monte-Carlo tests were conducted (25 runs) to investigate the effect of using the same undersampling masks for each contrast versus using different masks across contrasts.

An important concern regarding the use of joint regularization terms is leakage-of-features that are unique to a subset of the contrasts to other contrasts. To assess reliability against leakage-of-features, an elliptical dark region was introduced artificially in the PD-weighted image and an elliptical bright region was introduced in the T1-weighted image. These regions did not overlap spatially, and their intensities were accordingly set to the minimum and the maximum of their respective images. To increase the potential effect of unique features on the joint regularization terms, the dataset was reduced to three contrasts (PD-, T1-, T2-weighted). All acquisitions were 2D-accelerated with $R=4$.

To test stability against variations in the regularization parameters, all four parameters in SIMIT $\left(\alpha_{C T V}, \beta_{g L 1}, \gamma_{i T V}, \theta_{i L 1}\right)$ were individually scaled up/down until average SSIM across images (PD-, T1-, T2-weighted; 2D-acceleration, $R=3$ ) decreased from $98 \%$ (optimized parameters) to below $95 \%$. Since $\mu$ primarily controls convergence rate, it was not altered.

\section{7 | In-vivo data}

SIMIT was compared to Indiv-only and Joint-only as well as the state-of-the-art ESPIRiT method for retrospectively 2D-undersampled in-vivo data from $N=11$ participants. Methods were compared in terms of $\operatorname{pSNR}$ and $\operatorname{SSIM}(R=8, R=12, R=16)$ as well as via neuroradiologist reader studies $(R=8)$. Images reconstructed with ESPIRiT without any undersampling $(R=1)$ were used as reference in both evaluations. A neuroradiologist reader with 18 years of experience was blinded to method names, and methods were presented in randomized order. The reader evaluated the images for anatomical detail (1: low, 2: fair, 3: good/acceptable for clinical use, 4: very good, 5: excellent) as well as Gibbs artefacts and noise level (1: intolerable, 2: too much, 3: acceptable/not degrading the image, 4: very little, 5: none). All reference images were assigned a score of 5 in all categories by the reader to set a benchmark. Wilcoxon signed-rank test was performed on the reader scores as well as pSNR and SSIM measurements.

\section{8 | Further comparisons (supporting information)}

The proposed method was also compared to a collection of 6 state-of-the-art individual and joint reconstruction methods from the literature; Sparse MRI, ${ }^{25} \mathrm{TVCMRI}^{26}{ }^{2}$ RecPF, ${ }^{27} \mathrm{GSMRI}^{29}{ }^{29} \mathrm{FCS}^{28}$ and FCSA-MT ${ }^{31}$ using the numerical phantom and in-vivo data from a participant. Because these reference methods are for a single-coil receiver coil, these comparisons ${ }^{65}$ are given in the Supporting Information for conciseness.

\section{3 | RESULTS}

\section{$3.1 \quad$ Simulation results}

SIMIT consistently outperformed both Indiv-only and Joint-only in terms of SSIM for 1D- and 2D-acceleration, demonstrating the benefit of simultaneously using individual and joint regularization terms (Figure 3). On the average, SIMIT improved pSNR compared to Indiv-only and Jointonly by $1.7 \mathrm{~dB}$ and $4 \mathrm{~dB}$ for $1 \mathrm{D}$-acceleration, and by $1.7 \mathrm{~dB}$ and $4.4 \mathrm{~dB}$ for 2D-acceleration, respectively. While the difference in SSIM is rather small for two-fold 1D-acceleration, the difference between the methods becomes evident as the acceleration factor increases, with SIMIT yielding $1.6 \%$ and 3.6\% better SSIM than Indiv-only and Joint-only, respectively, averaged across all 1D-acceleration factors shown. For the range of 2Dacceleration factors shown, SIMIT yields $2.1 \%$ and $5 \%$ better SSIM compared to Indiv-only and Joint-only, respectively.

Different contrasts have different PSNR and SSIM values for the same acceleration factors as pSNR and SSIM depend on image content (Figure SI-8 in Supporting Information). Nevertheless, regardless of their individual pSNR and SSIM levels, Figure 4 demonstrates that all contrasts benefit from joint reconstruction, and consistently across the examined range of acceleration factors between $R=4$ and $R=15$. The performance improvement with increasing number of contrasts is more noticeable for higher acceleration factors. Averaged across contrasts, SSIM and pSNR were $0.8 \%$ and $2 \mathrm{~dB}$ higher for 5 -contrast reconstruction than individual reconstruction for $R=4$. For lower acceleration rates, SSIM curves are saturated near $100 \%$ and pSNR performance is enhanced for greater number of contrasts. For higher accelerations, PSNR curves are relatively flat 
FIGURE 3 Simultaneous use of individual and joint regularization terms (SIMIT) improves image quality (in terms of pSNR and SSIM) over using only individual (Indiv-only) or only joint (Jointonly) regularization terms at all examined acceleration factors for both one-dimensional $\left(R_{1 D}=2\right.$ to 5$)$, and two-dimensional $\left(R_{2 D}=4\right.$ to 15) acceleration. SSIM and pSNR were averaged across contrasts
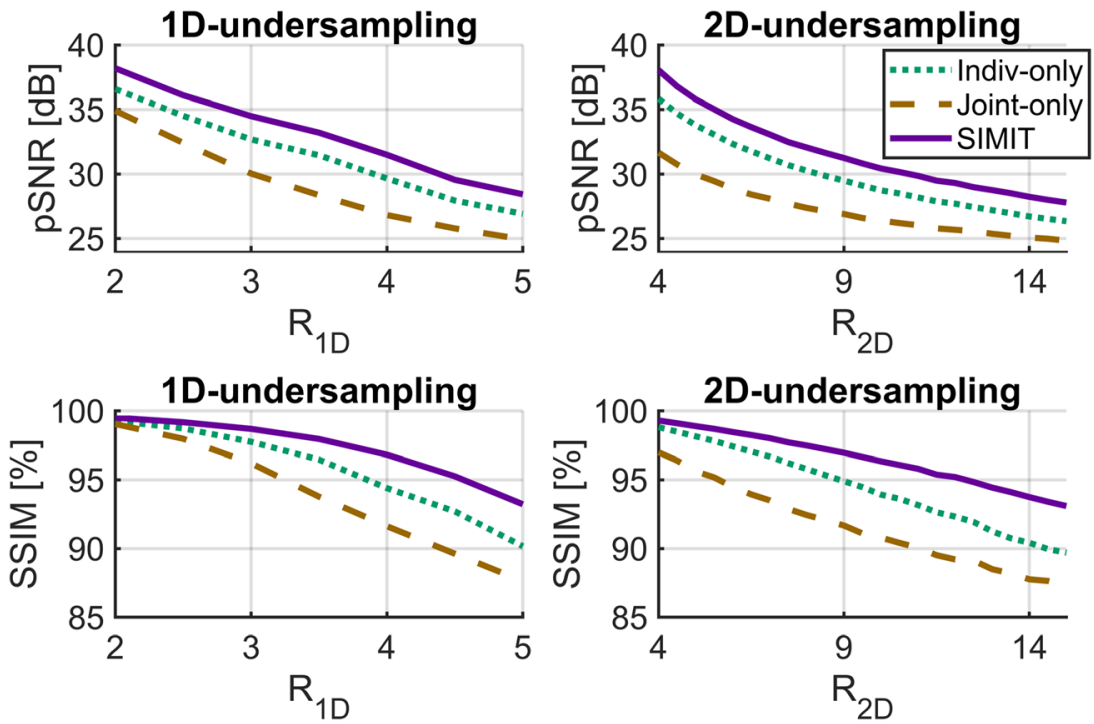

FIGURE 4 The variations of pSNR and SSIM with respect to the number of jointly reconstructed contrasts and the two-dimensional acceleration factor $\mathrm{R}$ is shown for SIMIT. As the number of contrasts that are jointly reconstructed increases, image quality increases for all contrasts and acceleration factors. For a given contrast and a given number of jointly reconstructed contrasts, pSNR and SSIM were averaged across all possible subsets of contrasts, e.g. for PD and 2-contrast reconstruction, SSIM of the PD-image was averaged across PD-T1, PD-T2, PD-STIR and PD-FLAIR reconstructions
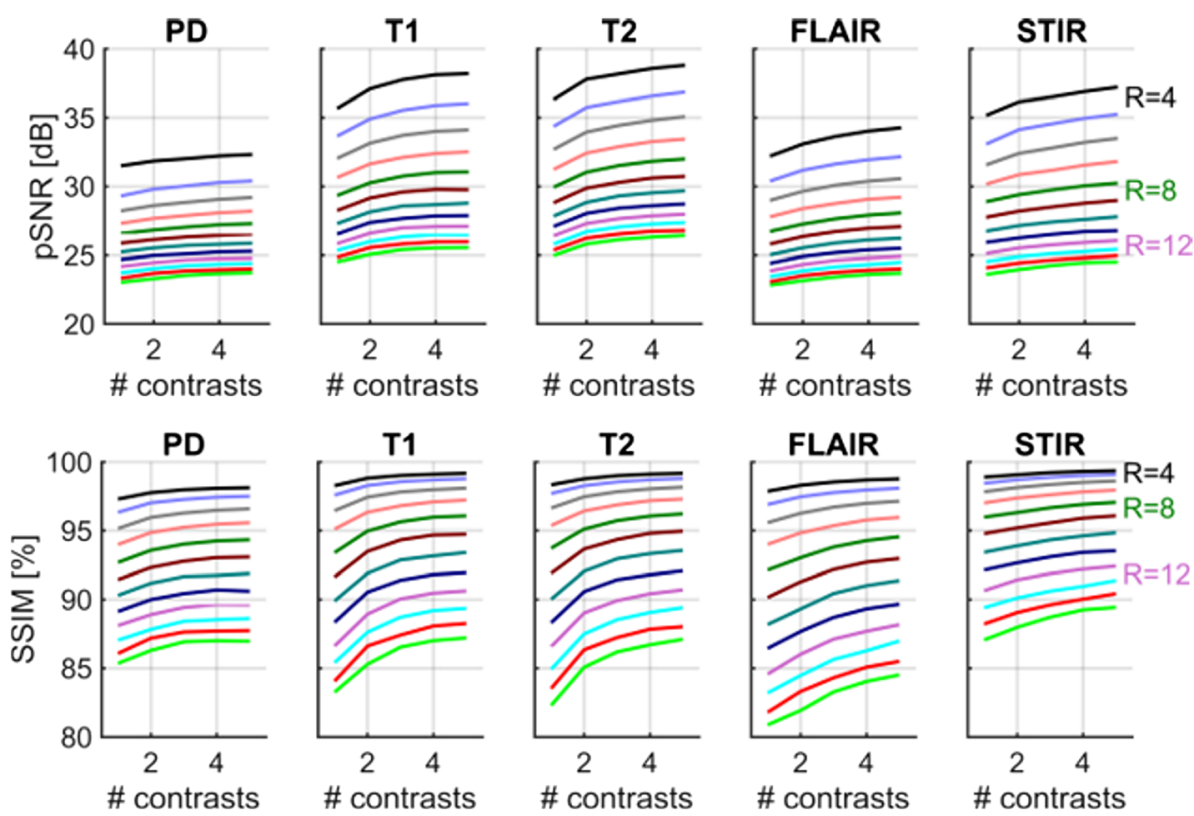

T2

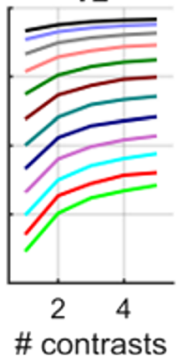

FLAIR

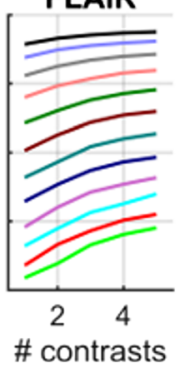

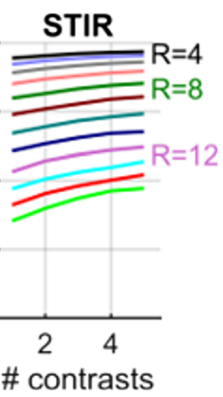

and SSIM performance is enhanced towards higher number of contrasts. Comparing a 5-contrast reconstruction to individual reconstruction, SSIM improvement monotonically increases to $3.3 \%$ SSIM at $R=15$, while the pSNR improvement monotonically decreases to $1 \mathrm{~dB}$ at $R=15$.

When identical undersampling masks were used for all contrasts instead of different masks across contrasts, the pSNR values dropped by only up to $0.1 \mathrm{~dB}$ across contrasts (data not shown). Therefore, the effect of using different versus identical undersampling masks on the performance of the proposed method was not further considered.

Despite increases in computation time per iteration with more regularization terms in SIMIT, the simultaneous use of individual and joint regularization terms in SIMIT enables improved reconstruction performance. Figure 5 shows the variations of pSNR and SSIM with respect to reconstruction time, and variations in undersampling masks and noise instances. SIMIT quickly surpasses Indiv-only and Joint-only to yield higher pSNR and SSIM before Indiv-only and Joint-only can converge to their final images. Note that these computation times exclude any parallel computation. Figure $5 \mathrm{~b}$ shows the change in SSIM and pSNR due to variations in noise and masks. SIMIT is less sensitive to these variations, yielding similar or lower standard variation across the Monte-Carlo runs.

Figure 6 visually compares SIMIT, Indiv-only and Joint-only in terms of leakage-of-features and reconstruction artefacts. Although Indiv-only does not have any leakage since images are reconstructed separately, the images suffer from residual noise-like artefacts due to undersampling. Joint-only reduces these undersampling artefacts but suffers from two potential drawbacks of joint reconstruction; leakage-of-features are 


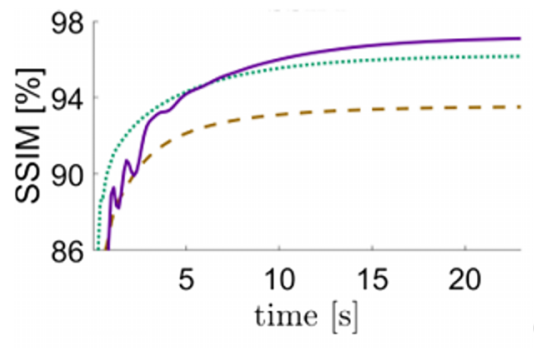

98

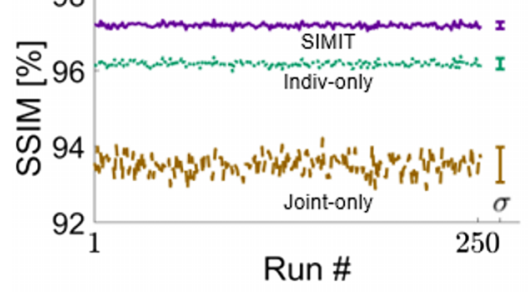

(B)

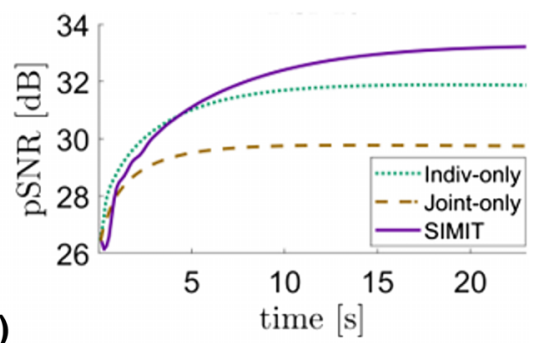

34

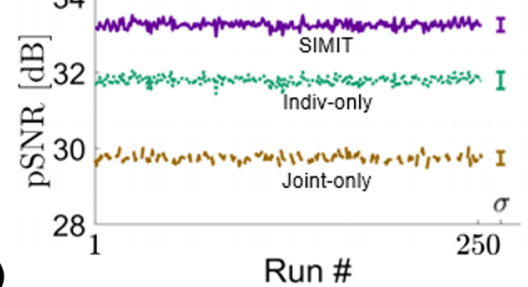

FIGURE 5 The variations of $\mathrm{pSNR}$ and SSIM with respect to (a) the reconstruction time and (b) variations in noise instances and undersampling masks. SSIM and pSNR were averaged across contrasts. In panel (a), SSIM and pSNR were averaged over 250 Monte-Carlo runs. Although SIMIT is mildly slower in improving image quality metrics in the first iterations, it quickly surpasses Indiv-only and Joint-only, yielding higher quality images before the metrics can reach steady-state for Indiv-only and Joint-only. In (b), SIMIT shows similar or better stability against variations in noise instances and undersampling masks. Standard deviation values are Indiv-only: 0.14\% and $0.22 \mathrm{~dB}$, Joint-only: $0.46 \%$ and $0.17 \mathrm{~dB}$, SIMIT: $0.09 \%$ and $0.17 \mathrm{~dB}$ for SSIM and pSNR, respectively

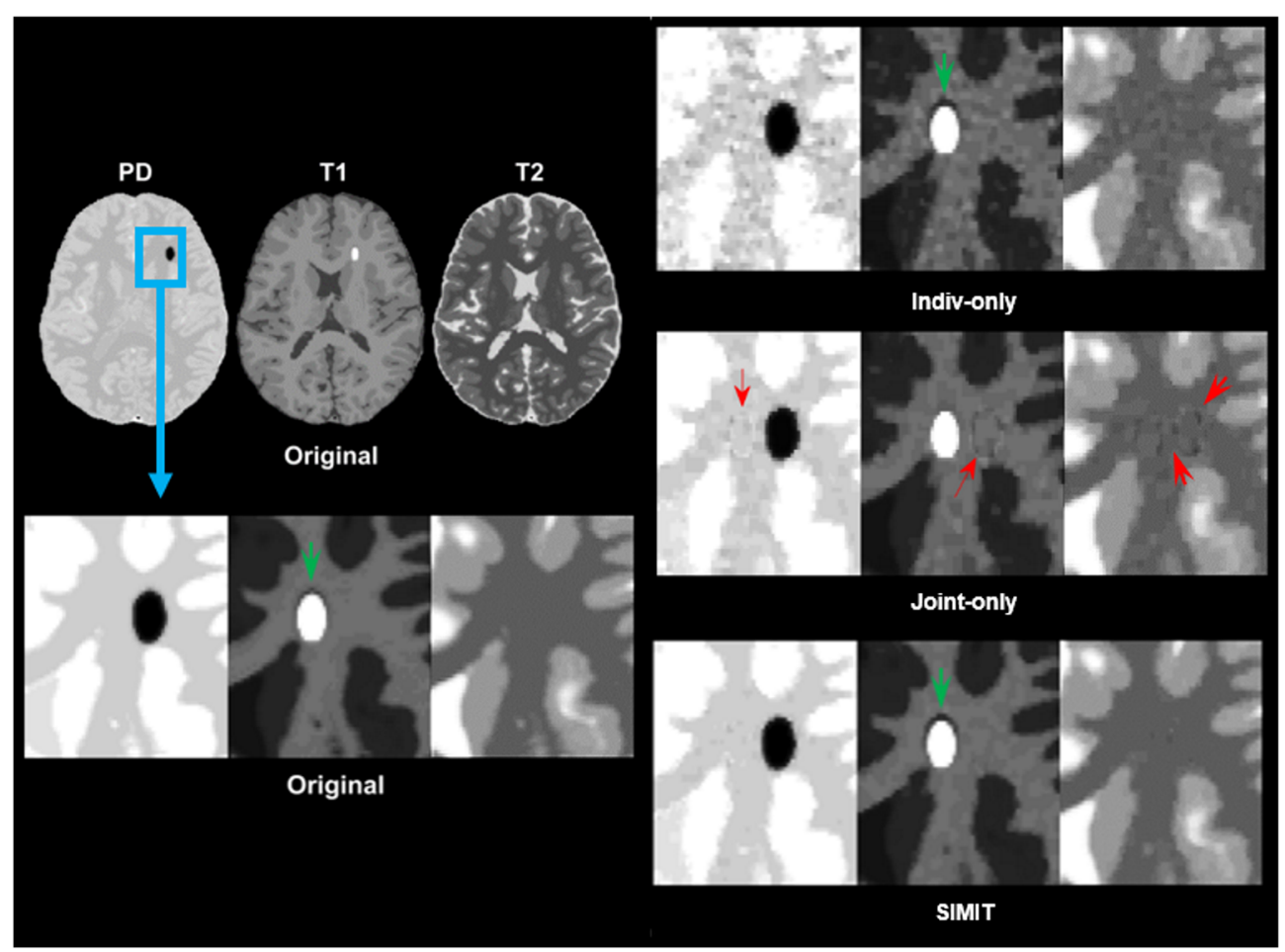

FIGURE 6 SIMIT, Indiv-only and Joint-only were compared in terms of leakage-of-features across contrasts. The numerical phantom was stripped of the skull and the skin for visualization purposes (but both tissues were included in the simulations). Red arrows show the leakage-offeatures in Joint-only reconstruction, which are suppressed with SIMIT. Green arrow shows that the black crescent seen in the original image is blurred in Joint-only, but it is clearly delineated with SIMIT

apparent in all images (red arrows) and blurring of unique features are seen in the dark crescent in the original T1-weighted image (green arrow). SIMIT does not have any structured leakage-of-features, and the intensity of the image reconstruction artefacts due to undersampling are below those observed for Indiv-only and Joint-only. Furthermore, the dark crescent is as clearly represented as in Indiv-only. These results indicate that the simultaneous use of individual and joint terms prevent both potential pitfalls of joint reconstruction. SIMIT also alleviates the staircase artefacts seen for Joint-only and suppresses the noise-like artefacts seen for Indiv-only.

With optimized regularization parameters, SIMIT yields an average SSIM of 98.1\% for the 3-fold 2D-accelerated three-contrast dataset. Individually scaling down $\alpha_{C T V}, \gamma_{i T V}, \beta_{g L 1}, \theta_{i L 1}$ up to an order of magnitude did not reduce SSIM below 95\%. Scaling the parameters up makes the regularization functions penalize the reconstruction more heavily and may lead to suboptimal reconstruction performance. SSIM still remained above 95\% when $\alpha_{C T V}, \gamma_{i T V}$ and $\theta_{i L 1}$ were individually scaled up by an order-of-magnitude. However, although SSIM remained above $95 \%$ when $\beta_{g L 1}$ was scaled up 5-fold, it reduced below 95\% when $\beta_{g L 1}$ was further doubled. All parameters except $\beta_{g L 1}$ have an order-of-magnitude headroom downwards and upwards, before noticeably affecting the image quality. 


\section{2 | In-vivo results}

SIMIT, Indiv-only and Joint-only as well as ESPIRiT were compared on in-vivo multi-channel acquisitions from $N=11$ participants. Magnified regions-of-interest (ROI) from representative reconstructions are shown in Figure 7 for PD-, T1- and T2-weighted images at $R=8$. Visual comparisons show that with respect to Indiv-only and Joint-only, which suffer from residual reconstruction error and noise, SIMIT yields better noise suppression and leads to a clearer depiction of tissues, particularly manifested inside the Lentiform Nucleus and the Putamen in the higher-SNR PDand T2-weighted images (Figures 7a and 7c). Furthermore, the grey-matter and white-matter boundaries are hard to distinguish in the lower-SNR T1-weighted image for Indiv-only and Joint-only, whereas SIMIT yields a clear depiction of these boundaries (Figure 7b). ESPIRiT also yields better noise suppression than Indiv-only and Joint-only, albeit at the cost of blurring and Gibbs-artefacts. Compared to ESPIRiT, SIMIT yields sharper images with better suppression of Gibbs-artefacts. This leads to a more accurate representation of the Globus Pallidus in the PD-weighted image, better delineation of the grey- and white-matter boundaries in the T1-weighted image, and the Putamen in the T2-weighted image.

Indiv-only and Joint-only show elevated levels of noise-like error while ESPIRiT and SIMIT yield improved suppression of noise-like artefacts as demonstrated by the error images between the reconstructions and the ideal reference of a representative participant in Figure $8(R=8)$. While the intensities of the error images are similar for ESPIRiT and SIMIT in the lower-SNR T1-weighted image, SIMIT outperforms ESPIRiT in artefact suppression for the higher-SNR PD- and T2-weighted images.

The error images were summed across all participants and contrasts to compare the methods for different acceleration factors (Figure 9). The reconstruction artefacts for SIMIT are visually less intense for all acceleration factors. Sparse reconstructions contain both noise-like and structured artefacts due to undersampling. In visualization of reconstructed images, the reduced intensity of noise-like artefacts in SIMIT might give the impression that structured artefacts are more prominent compared to Indiv-only and Joint-only, even though the latter methods also have similar levels of structured artefacts. To investigate this issue, we calculated the error images between each reconstructed image and the fullysampled reference image. The error images for separate reconstruction methods were then subtracted from each other to demonstrate potential differences in artefacts (Figure 10). The difference images have only noise-like behaviour and lack any structural information. This confirms that Indiv-only and Joint-only have similar levels of structured artefacts as SIMIT, but these are overshadowed by the higher levels of noise-like error in Indiv-only and Joint-only.

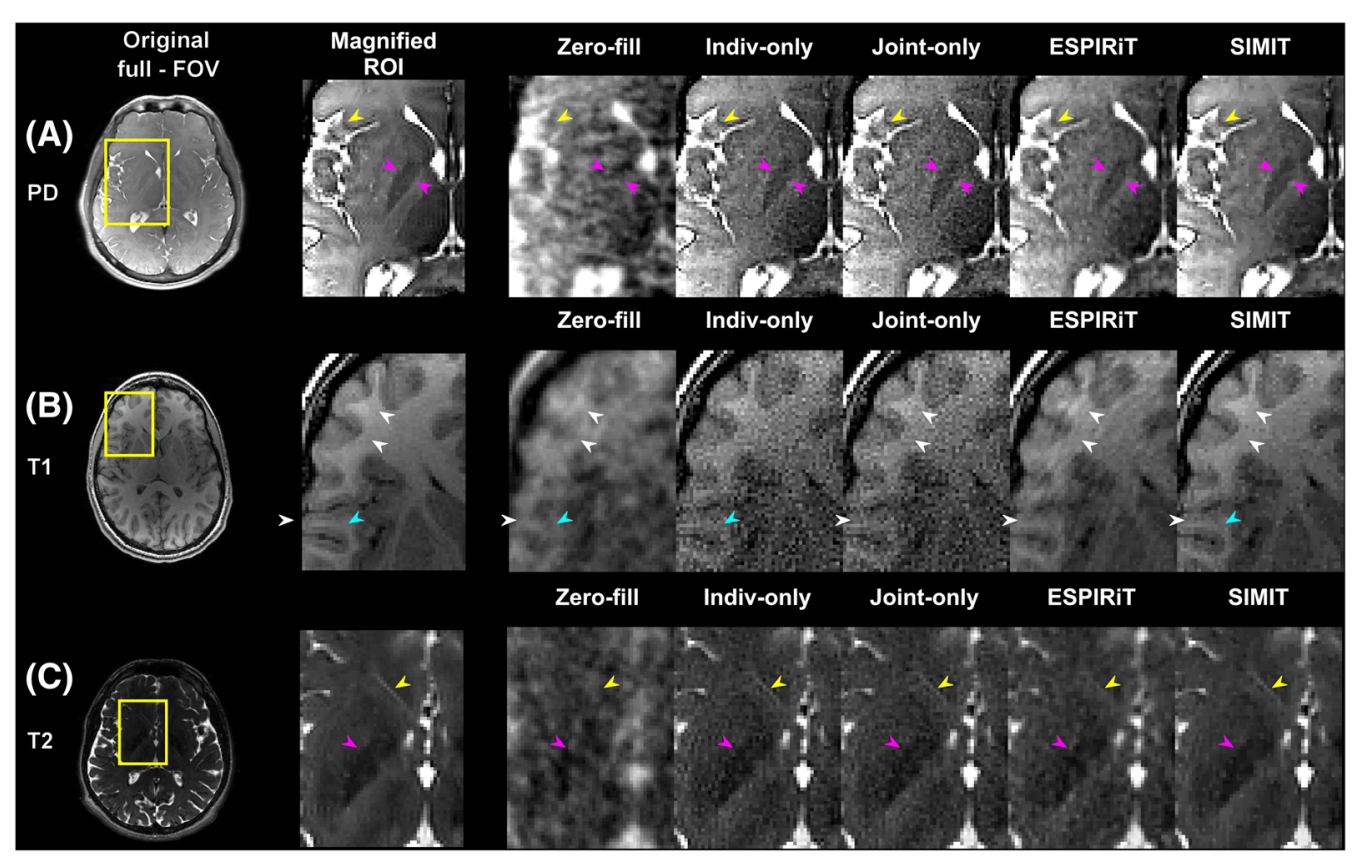

FIGURE 7 Representative reconstructions of (a) PD-weighted, (b) T1-weighted, (c) T2-weighted images from three different participants for all methods at $R=8$. Magnified views show the regions bounded by the yellow rectangles. (a) Indiv-only and Joint-only suffer from noise, while ESPIRiT shows blurring at the boundary of the frontal opercular cortex (yellow arrows) and a narrower representation of the Globus Pallidus (pink arrows). SIMIT yields better noise suppression while demonstrating a clearer delineation of tissue at the frontal opercular cortex (yellow arrow) and inside the Lentiform nucleus (pink arrows). (b) Due to the relatively lower SNR of the T1-weighted image, grey-matter boundaries in the sulci cannot be identified in Indiv-only and Joint-only reconstructions (cyan arrow). For ESPIRiT, while noise suppression is much better, tissue delineation is compromised in the gyri due to the Gibbs-like artefacts (white arrows). SIMIT yields much better suppression than Indiv-only and Joint-only while yielding a clear depiction of grey-matter white-matter boundaries without Gibbs-artefacts. (c) SIMIT yields better delineation of the putamen (pink arrow) as well as the partial volume of the lateral ventricle (yellow arrow) compared to the other methods 


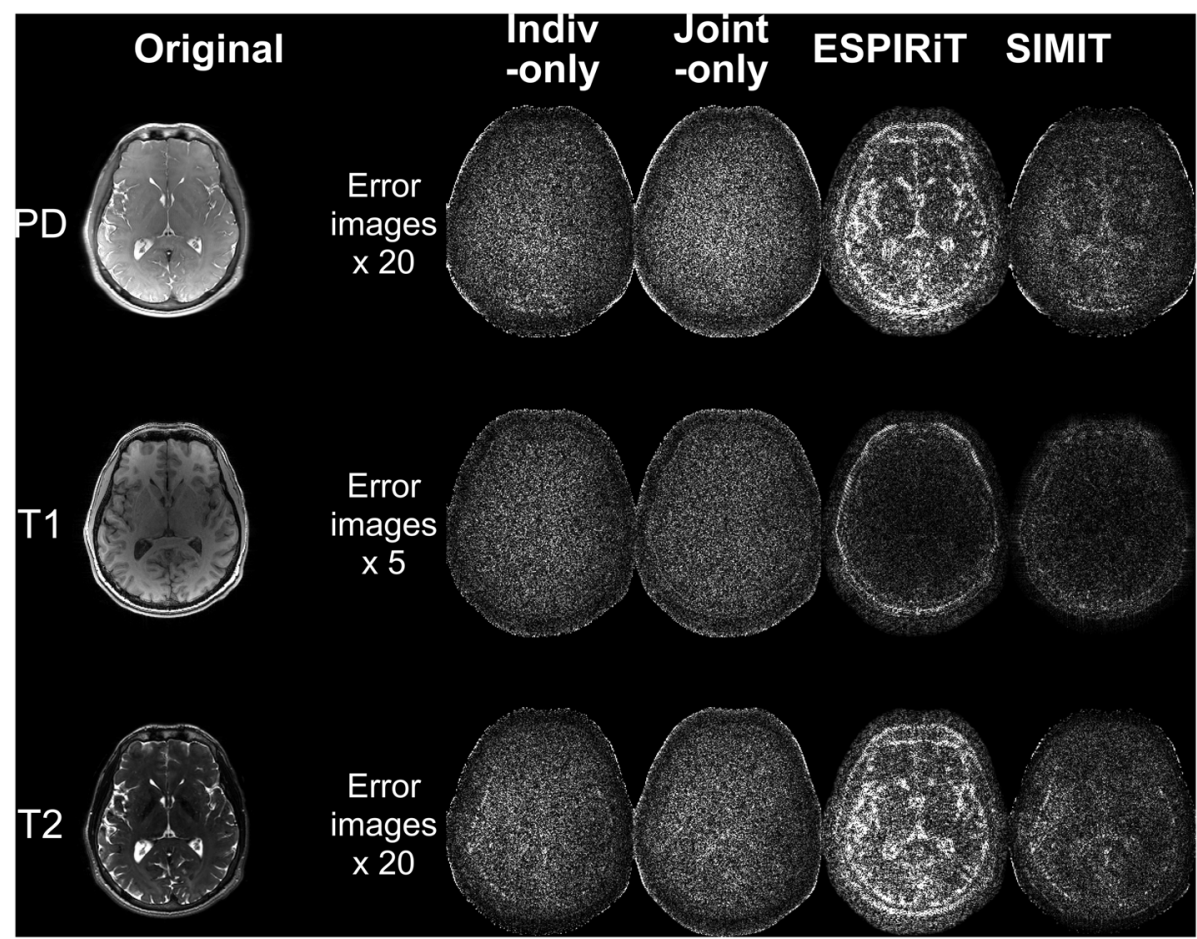

FIGURE 8 Error images were calculated between the fully-sampled reference image and the reconstructed images for all methods at $R=8$. Indiv-only and Joint-only suffer from noise-like reconstruction artefacts for all contrasts. For the lower-SNR T1-weighted image, the error images for ESPIRiT and SIMIT have similar intensity, although the error for ESPIRiT is considerably more intense than SIMIT for PD- and T2-weighted images

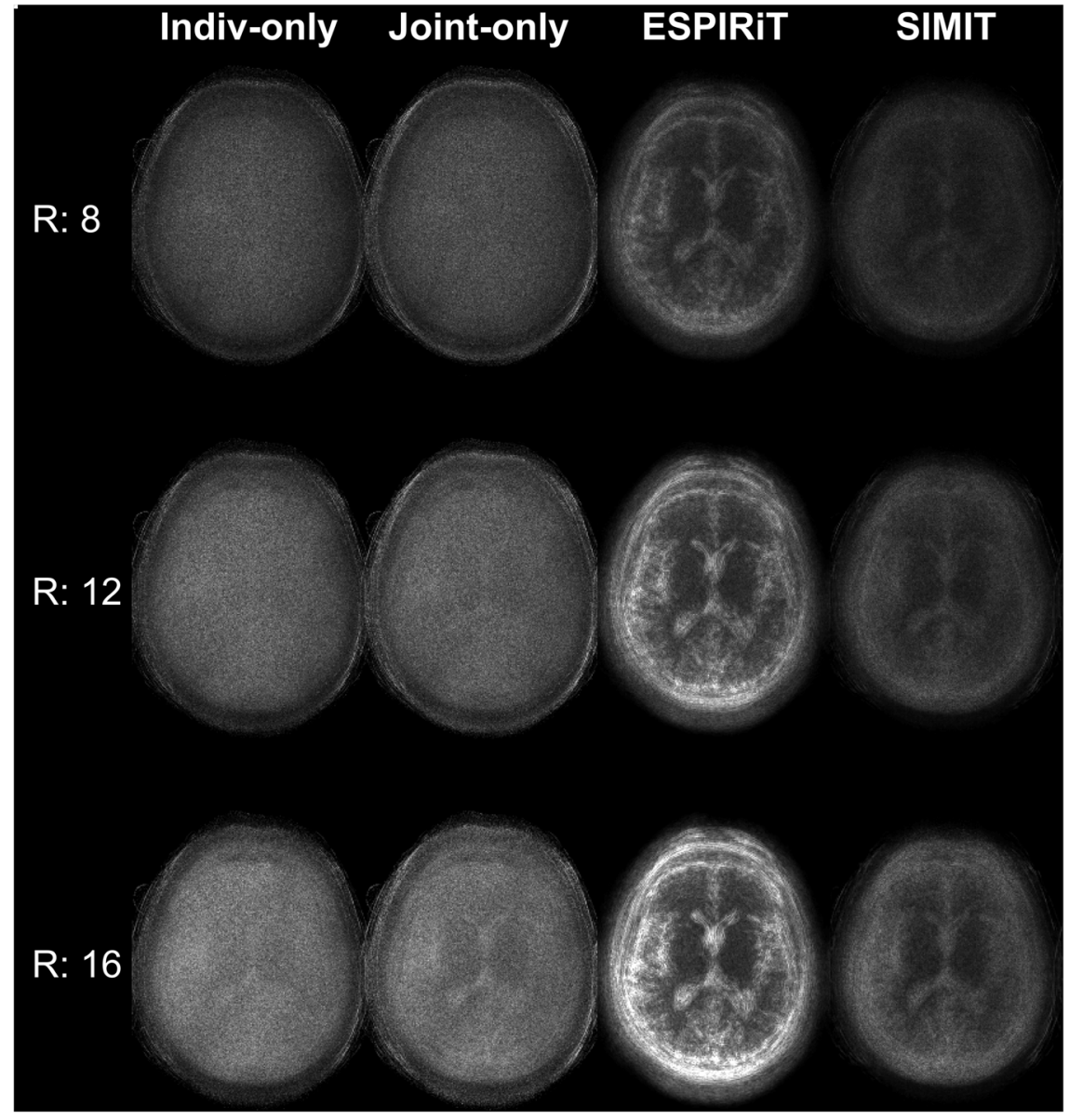

FIGURE 9 Maps of reconstruction error were calculated for each contrast in each individual subject. Error maps were averaged across all contrasts and participants. Error maps were intensified 10 -fold and shown in the same colour-scale as the originals in Figure 8. Error maps are shown for all methods at $\mathrm{R}=8, R=12$ and $R=16$. On average, SIMIT yields visually reduced reconstruction artefacts compared to reference methods 
FIGURE 10 The difference images for SIMIT were subtracted from the difference images for Indiv-only and Joint-only, and then summed over $N=11$ participants. The resulting maps show noise-like behaviour but lack structural information. This demonstrates that structured artefacts are at similar levels for SIMIT, Indiv-only and Joint-only

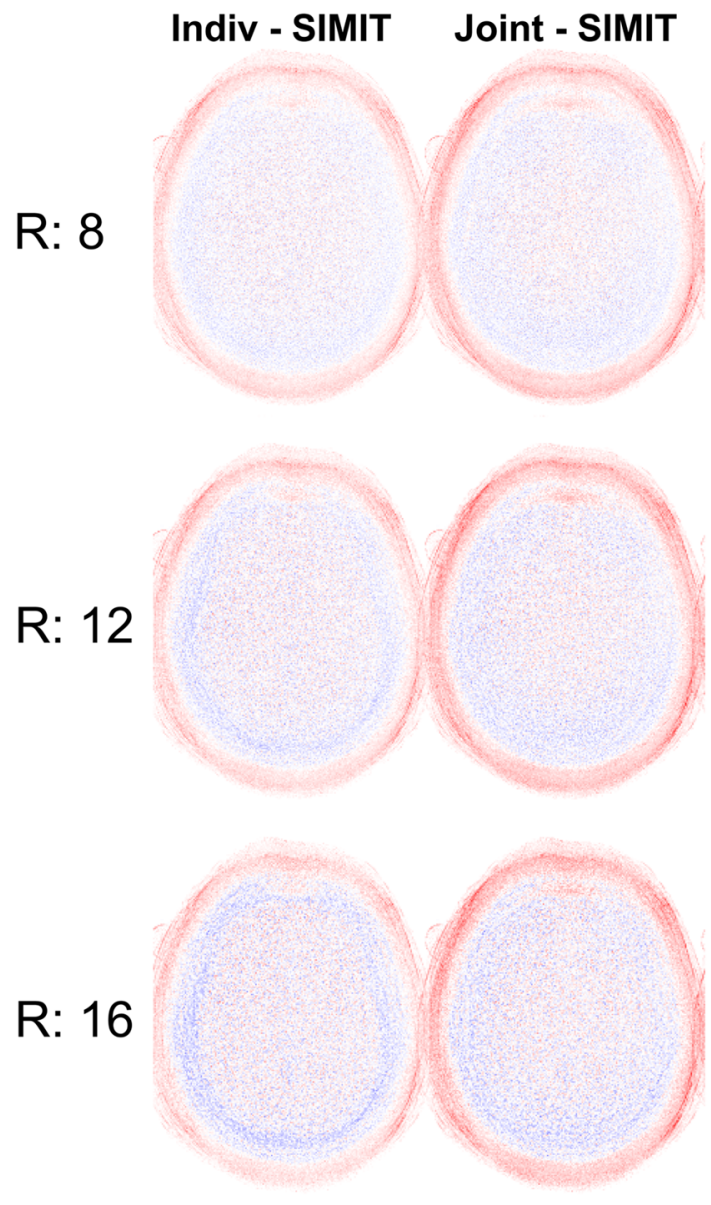

Methods were also compared quantitatively. Statistical analysis showed that SIMIT yields significantly better pSNR and SSIM values for all contrasts and acceleration factors $(R=8, R=12$ and $R=16)$ compared to all methods $(p<0.05$, Figure 11). Averaged across participants, contrasts and acceleration factors, SIMIT yielded higher pSNR values than Indiv-only, Joint-only and ESPIRiT by $4.5 \mathrm{~dB}, 5.0 \mathrm{~dB}$ and $4.3 \mathrm{~dB}$ at $\mathrm{R}=8$; by $4.1 \mathrm{~dB}, 4.4 \mathrm{~dB}$ and $6.0 \mathrm{~dB}$ at $\mathrm{R}=12$; and by $3.6 \mathrm{~dB}, 3.7 \mathrm{~dB}$ and $6.5 \mathrm{~dB}$ at $\mathrm{R}=16$, respectively. Compared to all reference methods across all acceleration factors, SIMIT yielded at least $3.6 \mathrm{~dB}$ improvement in pSNR.

ESPIRiT yielded relatively more consistent reconstruction performance across contrasts, with less than $3 \mathrm{~dB}$ variation in (participant-averaged) pSNR across contrasts for all acceleration factors. For Indiv-only and Joint-only, the variation across contrasts was as high as $10 \mathrm{~dB}$. SIMIT yielded more consistent results than Indiv-only and Joint-only with up to $6 \mathrm{~dB}$ pSNR variation across contrasts. Even though the variation was larger than that of ESPIRiT, comparing the maximum pSNR values of ESPIRiT (blue triangular markers) and the minimum pSNR values of SIMIT (red triangular markers) shows that the pSNR values of SIMIT were higher than those of ESPIRiT for all contrasts, acceleration factors and participants.

Joint reconstruction via SIMIT allows increasing the acceleration factor without compromising image quality. For the PD-weighted image, SIMIT allows increasing $R=8$ to $R=12$ compared to Indiv-only and ESPIRiT, and $R=16$ compared to Joint-only while improving pSNR and SSIM. For the T1-weighted image, $R=8$ can be increased to $R=10$ (not shown) compared to ESPIRiT and $R=16$ compared to Indiv-only and Joint-only with better pSNR and SSIM. For the T2-weighted image, SIMIT yields better pSNR and SSIM at R $=10$ than Indiv-only and Joint-only at $R=8$, and at $\mathrm{R}=16$ than ESPIRiT at $R=8$.

To confirm that the visual and quantitative improvements in image quality enabled by SIMIT translate to diagnostic assessment, neuroradiologist reader studies were conducted for $\mathrm{R}=8$ (Figure 12). For all contrasts and comparisons in terms of anatomy, noise and Gibbs-artefacts, SIMIT yields higher scores than the other methods. SIMIT yields significantly better $(p<0.05)$ anatomy scores than the other methods except for T1-weighted against ESPIRiT, where the two methods perform similarly. In terms of noise, SIMIT performs significantly better than Indiv-only and ESPIRiT for two of the contrasts while performing similarly for a third, and it performs significantly better than Joint-only for all contrasts. In terms of Gibbs artefacts, SIMIT performs significantly better than all other methods for PD-weighted images. SIMIT performs significantly better than ESPIRiT for T1-weighted images, while performing similarly to Indiv-only and Joint-only. Meanwhile, all methods perform similarly for T2-weighted images. For each contrast, SIMIT yields significantly better scores $(p<0.05)$ in at least one of the comparisons (anatomy/noise/Gibbs) against each alternative method. 

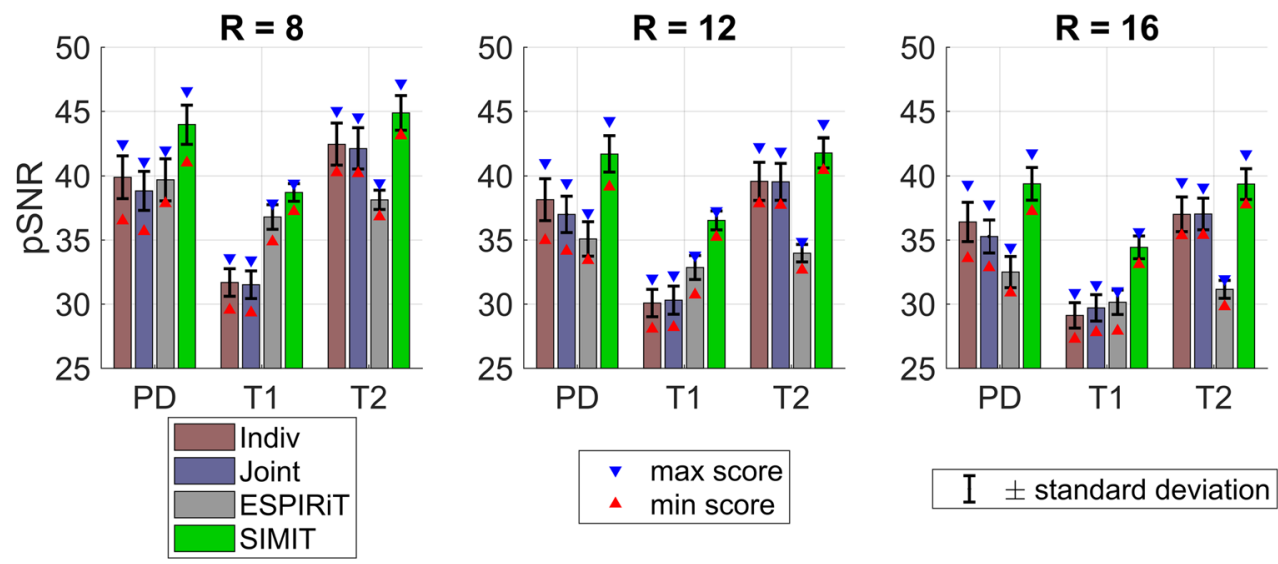

FIGURE 11 Methods are compared in terms of pSNR and SSIM for all participants and contrasts at $R=8, R=12$ and $\mathrm{R}=16$. SIMIT yields significantly higher pSNR and SSIM $(p<0.05)$ than all methods, consistently across acceleration factors and contrasts. Blue and red arrows show the maximum and minimum values, respectively, and the error bars show the standard deviation . max score
. $\min$ score I \pm standard deviation of the measured metric (pSNR or SSIM)
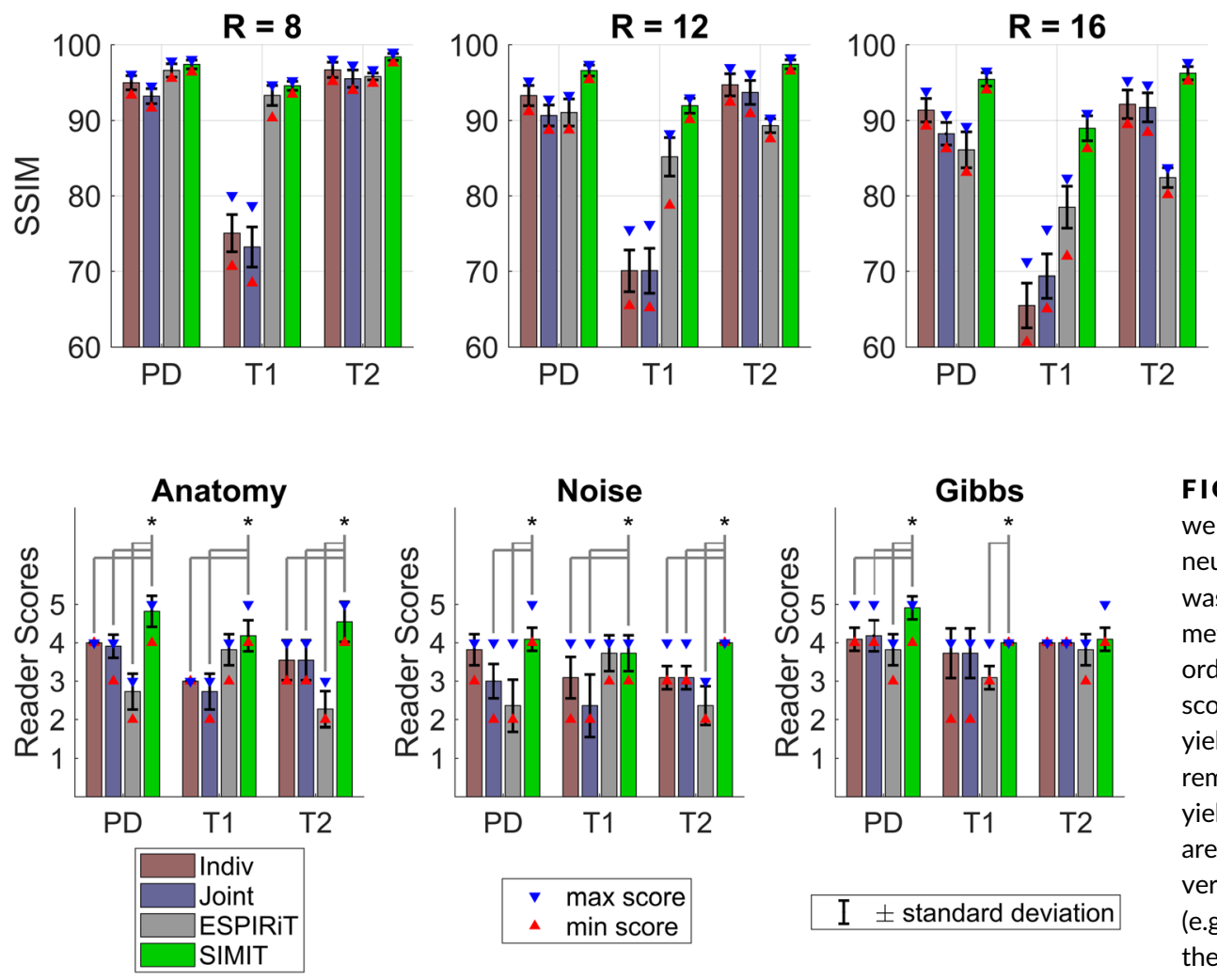

FIGURE 12 Reconstruction methods were compared in terms of neuroradiologist reader scores. The reader was blinded to method names and methods were presented in randomized order. SIMIT yields significantly higher scores in 19 out of 27 comparisons and yields similar performance in the remaining cases. The methods SIMIT yields significantly higher scores against are indicated by the asterisks and the vertical bars below the asterisks (e.g. against Joint-only and ESPIRiT for the T1-weighted image in terms of noiselevel). Blue and red arrows show the maximum and minimum scores, respectively, and the error bars show the standard deviation of the scores

SIMIT performs significantly better $(p<0.05)$ than Indiv-only, Joint-only and ESPIRiT in six, seven and six out of nine comparisons (three contrasts, anatomy/noise/Gibbs), respectively. Averaged across participants, contrasts and categories, the neuroradiologist scores for SIMIT were higher by $0.7,0.9$ and 1.2 compared to Indiv-only, Joint-only and ESPIRiT, respectively.

\subsection{Further Comparisons (Supporting Information)}

SIMIT was also compared to a large collection of state-of-the-art methods from the literature ${ }^{25-29,31,59}$ in terms of pSNR, SSIM and computation speed (Supporting Information). While the reference method RecPF had a faster initial improvement in pSNR and SSIM, SIMIT quickly surpassed RecPF and converged to higher quality images (Figure SI-2). SIMIT was also more robust to variations in undersampling masks and noise 
(Figure SI-2), yielded better suppression of reconstruction artefacts (Figures SI-3 and SI-4), and provided better SSIM and pSNR for various 1D- and 2D-acceleration rates (Figure SI-5). Finally, SIMIT yielded a clearer and sharper depiction of tissues for the in-vivo data (Figures SI-6 and SI-7).

\section{4 | DISCUSSION}

The proposed multi-channel multi-acquisition reconstruction method, SIMIT, incorporates both joint and individual regularization terms across multi-contrast images. The complex optimization problem that arises is solved via the ADMM algorithm. SIMIT enhances sparse recovery for multi-contrast datasets, for both single- and multi-channel receiver coils. It also enables prescription of higher acceleration factors through joint reconstruction of multi-contrast acquisitions. In multi-contrast reconstructions, SIMIT outperforms a variant method that only includes individual regularization terms (Indiv-only), a variant that only includes joint regularization terms (Joint-only), as well as a state-of-the-art parallel imaging method (ESPIRiT). Compared to Indiv-only and Joint-only, SIMIT lowers reconstructions errors due to residual noise and aliasing. While Joint-only suffers from visible feature leakage across contrast, SIMIT yields enhanced reliability against these artefacts. SIMIT also improves recovery of high spatial frequency details compared to ESPIRiT. The enhanced image quality of SIMIT is also apparent in both quantitative metrics and neuroradiologist reader scores.

Even though the proposed method uses four regularization terms, the additional time required by using more regularization terms is relatively small compared to the time required to complete a whole iteration. Furthermore, using all terms simultaneously improves image quality with respect to individual-only and joint-only reconstruction due to information sharing and prevention of leakage-of-features, respectively. Therefore, image quality rapidly improves in fewer iterations, and the method converges to higher-quality images.

In this study, non-identical undersampling masks were used for each contrast. Even though random undersampling patterns in CS lead to incoherent undersampling artefacts, using identical masks for each contrast may create a coherence in the undersampling artefacts across contrasts. The method was also tested with identical undersampling masks across contrasts, however, the difference with respect to using nonidentical masks was modest. This could partly be attributed to the dissimilarity of the jointly reconstructed contrasts. Even though the undersampled frequencies are the same, the energy content at these frequencies is different for each contrast, leading to dissimilar undersampling artefacts. Using identical undersampling masks could potentially lead to further reductions in imaging performance for similar contrasts such as multi-echo acquisitions at different echo-times. Detailed comparison of using identical undersampling masks to undersampling masks explicitly designed to complement each other can be found in Refs. ${ }^{43,70}$

Previous joint reconstruction approaches in MRI include using nuclear and Frobenius norms ${ }^{35,36}$ for dynamic MRI; K-SVD ${ }^{71}$ for parametric mapping, ${ }^{37}$ minimizing the sum of individual regularization functions, ${ }^{38}$ spatially weighting the regularization terms of an image using a prior image for multi-contrast MRI, ${ }^{49}$ and replacing one or both of the $e_{1}$-sparsity and TV terms with group sparsity and CTV for diffusion tensor imaging, ${ }^{39}$ parametric mapping, ${ }^{40}$ multi-echo T2-weighted imaging ${ }^{29,33}$ and multi-contrast imaging. ${ }^{30,31}$ In this study, our choice in regularization terms was motivated by two reasons. First, we preferred the more commonly used $\ell_{1}$-sparsity and Total Variation to other alternatives, since specific terms used in dynamic MRI and parametric mapping may not be directly applicable to multi-contrast datasets that comprise a small number of static acquisitions under distinct contrasts, and that may not lead to an overcomplete dictionary suitable for K-SVD. Second, we simultaneously used individual and joint versions of the regularization terms to create a balance between utilizing common features across images and preserving individual features of each contrast. Group $e_{1}$-sparsity was introduced for improving signal recovery in low-SNR voxels, in cases where the signal is present broadly across contrasts. Note that group-sparsity can also lead to unwanted suppression of a signal that is present only in a small subset of contrasts. For such cases, individual sparsity was introduced to retain contrast-specific signals. Similarly, Colour TV better distinguishes tissue boundaries in lower-SNR images when there is clear delineation of tissues in a higher-SNR image. Any possible detrimental effects, when all images except one have noisy patterns in smoothly varying regions or across tissue boundaries, were prevented by the individual TV as it serves to reduce noise in individual images.

In practice, individual reconstruction of each acquisition in a multi-contrast protocol is better suited to online processing as it improves workflow by recovering the images for the a given contrast while data are being acquired for the next contrast. However, this does not preclude a workflow in which a given contrast is reconstructed without latency as the data become available to guide the prescription of later acquisitions in the protocol. At the end of the protocol, all acquisitions can still be jointly reconstructed for maximal image quality. This workflow would be similar to the one in Ref.49 with the difference being that in SIMIT both images are jointly reconstructed instead of using an initially reconstructed image to improve the reconstruction of later images without updating the first.

Selection of regularization parameters has a critical effect on the convergence behaviour and resultant image quality of regularized reconstructions. Each of the four regularization parameters used in SIMIT were separately varied until the average SSIM was reduced from $98 \%$ to below $95 \%$. No significant variations in image quality were observed when the parameters were scaled up or down by an order of magnitude, suggesting that SIMIT is reasonably robust against variations in reconstruction parameters. The most sensitive parameter was that of group sparsity while the other parameters had broader margins. The optimal parameters may show larger deviations for body parts with substantially 
different tissue structure (e.g., the abdomen versus the brain). In such cases, parameters can be optimized a priori on a training dataset based on the anatomy of interest, yielding anatomy-specific sets of parameters.

The relative scales of image intensities and regularization parameters can affect the progression of iterative reconstructions. In case of a large mismatch in scale, it was observed that the updates in each iteration were either excessively small or large in magnitude, which caused all methods tested here to result in poor reconstructions. Therefore, intensity normalization was used to improve image quality and to ensure that similar ranges of regularization parameters work well across datasets. This is particularly important for joint reconstruction of multiple contrasts since image scales may vary significantly across acquisitions, and acquisitions with higher image intensities can dominate calculations of joint regularization terms such as joint sparsity or colour TV. To prevent potential scale-related biases, k-space data for each acquisition were normalized in this study, such that the respective fully-sampled reconstructed images are in the same range. Assuming similar initial noise levels, this normalization scales the noise-level for images with relatively low intensity upward, compared with the noise-floor of the images with higher intensity. To compensate for this increase in the noise level, we had to adjust the individual regularization terms for the T1-weighted image for all methods that use individual regularization to improve image quality. Even prior to adjustment, we prefer imbalanced noise levels across acquisitions to poor reconstruction quality.

SIMIT was demonstrated with both 1D and 2D undersampling, and thus it can be applied to both 2D and 3D imaging. Here we applied the regularization terms on cross-sectional images across the phase-encode directions. Alternatively, an entirely 3D optimization problem can be cast with regularization terms also incorporating tissue information along the readout dimension. In that case, group sparsity terms can be enforced across multiple cross-sections to further improve reconstruction performance.

\section{ACKNOWLEDGEMENTS}

This work was supported in part by Turkish Scientific and Technological Research Council (TÜBiTAK) grant with Project \#3151068, by a European Molecular Biology Organization Installation Grant (IG 3028), by Turkish Academy of Sciences (TUBA) GEBIP 2015 fellowships, and by BAGEP awards by The Science Academy, Turkey.

\section{ORCID}

Emre Kopanoglu (D) https://orcid.org/0000-0001-8982-4441

Alper Güngör (D) https://orcid.org/0000-0002-3043-9124

Toygan Kilic (D) https://orcid.org/0000-0001-7969-5738

Emine Ulku Saritas (D) https://orcid.org/0000-0001-8551-1077

Kader K. Oguz (D) https://orcid.org/0000-0002-6404-4403

Tolga Çukur (D) https://orcid.org/0000-0002-2296-851X

H. Emre Güven (D) https://orcid.org/0000-0001-5665-8782

\section{REFERENCES}

1. Sodickson DK, Manning WJ. Simultaneous acquisition of spatial harmonics (SMASH): fast imaging with radiofrequency coil arrays. Magn Reson Med. 1997;38(4):591-603.

2. Pruessmann KP, Weiger M, Scheidegger MB, Boesiger P. SENSE: sensitivity encoding for fast MRI. Magn Reson Med. 1999;42(5):952-962.

3. Griswold MA, Jakob PM, Heidemann RM, et al. Generalized autocalibrating partially parallel acquisitions (GRAPPA). Magn Reson Med. 2002;47(6): 1202-1210.

4. Blaimer M, Breuer F, Mueller M, Heidemann RM, Griswold MA, Jakob PM. SMASH, SENSE, PILS, GRAPPA: how to choose the optimal method. Top Magn Reson Imaging. 2004;15(4):223-236.

5. Breuer FA, Blaimer M, Heidemann RM, Mueller MF, Griswold MA, Jakob PM. Controlled aliasing in parallel imaging results in higher acceleration (CAIPIRINHA) for multi-slice imaging. Magn Reson Med. 2005;53(3):684-691.

6. Breuer FA, Blaimer M, Mueller MF, et al. Controlled aliasing in volumetric parallel imaging (2D CAIPIRINHA). Magn Reson Med. 2006;55(3):549-556.

7. Setsompop K, Cohen-Adad J, Gagoski BA, et al. Improving diffusion MRI using simultaneous multi-slice echo planar imaging. Neuroimage. 2012;63(1): 569-580.

8. Feinberg DA, Setsompop K. Ultra-fast MRI of the human brain with simultaneous multi-slice imaging. J Magn Reson. 2013;229(0):90-100.

9. Saritas EU, Lee D, Cukur T, Shankaranarayanan A, Nishimura DG. Hadamard slice encoding for reduced-FOV diffusion-weighted imaging. Magn Reson Med. 2014;72(5):1277-1290.

10. Feinberg DA, Hoenninger JC, Crooks LE, Kaufman L, Watts JC, Arakawa M. Inner volume MR imaging: technical concepts and their application. Radiology. 1985;156(3):743-747.

11. Bottomley PA, Hardy CJ. Two-dimensional spatially selective spin inversion and spin-Echo refocusing with a single nuclear-magnetic-resonance pulse. J Appl Phys. 1987;62(10):4284-4290.

12. Singh S, Rutt BK, Henkelman RM. Projection Presaturation - a fast and accurate technique for multidimensional spatial localization. J Magn Reson. 1990;87(3):567-583.

13. Pauly JM, Hu BS, Wang SJ, Nishimura DG, Macovski A. A three-dimensional spin-echo or inversion pulse. Magn Reson Med. 1993;29(1):2-6.

14. Morrell G, Macovski A. Three-dimensional spectral-spatial excitation. Magn Reson Med. 1997;37(3):378-386.

15. Lee SY, Cho ZH. Localized volume selection technique using an additional radial gradient coil. Magn Reson Med. 1989;12(1):56-63. 
16. Kopanoglu E, Constable RT. Radiofrequency pulse design using nonlinear gradient magnetic fields. Magn Reson Med. 2015;74(3):826-839.

17. Kopanoglu E, Atalar E, Constable RT. Reduced-FOV imaging with excitation using nonlinear gradient magnetic fields (ENiGMa). Paper presented at: proceedings of the 21st annual meeting of ISMRM2013; Salt Lake City, UT, USA

18. Witschey WR, Cocosco CA, Gallichan D, et al. Localization by nonlinear phase preparation and k-space trajectory design. Magn Reson Med. 2012; 67(6):1620-1632.

19. Malloy CR, Lange RA, Klein DL, et al. Spatial localization of Nmr signal with a passive surface gradient. J Magn Reson. 1988;80(2):364-369.

20. Layton KJ, Gallichan D, Testud F, et al. Single shot trajectory design for region-specific imaging using linear and nonlinear magnetic encoding fields. Magn Reson Med. 2013;70(3):684-696.

21. Kopanoglu E, Wang H, Wan Y, Peters DC, Galiana G, Constable RT. rOi-space: accelerated imaging of sub-volumes using ROI focused O-space. Paper presented at: proceedings of the 23rd annual meeting of ISMRM2015; Toronto, Cana

22. Saritas EU, Cunningham CH, Lee JH, Han ET, Nishimura DG. DWI of the spinal cord with reduced FOV single-shot EPI. Magn Reson Med. 2008;60(2): 468-473.

23. Candes EJ, Romberg J, Tao T. Robust uncertainty principles: exact signal reconstruction from highly incomplete frequency information. leee Transactions on Information Theory. 2006;52(2):489-509.

24. Donoho DL. Compressed sensing. IEEE Transactions on Information Theory. 2006;52(4):1289-1306.

25. Lustig M, Donoho D, Pauly JM. Sparse MRI: The application of compressed sensing for rapid MR imaging. Magn Reson Med 2007;58(6):1182-1195.

26. Shiqian M, Wotao Y, Yin Z, Chakraborty A. An efficient algorithm for compressed MR imaging using total variation and wavelets. Paper presented at: IEEE CVPR; 23-28 June 2008, 2008; Anchorage, Alaska, USA.

27. Yang JF, Zhang Y, Yin WT. A fast alternating direction method for TVL1-L2 signal reconstruction from partial Fourier data. leee Journal of Selected Topics in Signal Processing. 2010;4(2):288-297.

28. Huang J, Zhang S, Metaxas D. Efficient MR image reconstruction for compressed MR imaging. Med Image Anal. 2011;15(5):670-679.

29. Majumdar A, Ward RK. Joint reconstruction of multiecho MR images using correlated sparsity. Magn Reson Imaging. 2011;29(7):899-906.

30. Bilgic B, Goyal VK, Adalsteinsson E. Multi-contrast reconstruction with Bayesian compressed sensing. Magn Reson Med. 2011;66(6):1601-1615.

31. Huang J, Chen C, Axel L. Fast multi-contrast MRI reconstruction. Magn Reson Imaging. 2014;32(10):1344-1352.

32. Otazo R, Kim D, Axel L, Sodickson DK. Combination of compressed sensing and parallel imaging for highly accelerated first-pass cardiac perfusion MRI. Magn Reson Med. 2010;64(3):767-776.

33. Majumdar A, Ward RK. Accelerating multi-echo T2 weighted MR imaging: analysis prior group-sparse optimization. J Magn Reson. 2011;210(1):90-97.

34. Knoll F, Holler M, Koesters T, Otazo R, Bredies K, Sodickson DK. Joint MR-PET reconstruction using a Multi-Channel image Regularizer. IEEE Trans Med Imaging. 2017;36(1):1-16.

35. Lingala SG, Hu Y, DiBella E, Jacob M. Accelerated dynamic MRI exploiting Sparsity and low-rank structure: k-t SLR. IEEE Trans Med Imaging. 2011; 30(5):1042-1054.

36. Majumdar A, Ward R. Learning space-time dictionaries for blind compressed sensing dynamic MRI reconstruction. Paper presented at: 2015 IEEE International Conference on Image Processing (ICIP); 27-30 Sept. 2015, 2015.

37. Doneva M, Bornert P, Eggers H, Stehning C, Senegas J, Mertins A. Compressed sensing reconstruction for magnetic resonance parameter mapping. Magn Reson Med. 2010;64(4):1114-1120.

38. Doneva M, Bornert P, Eggers H, Mertins A, Pauly J, Lustig M. Compressed sensing for chemical shift-based water-fat separation. Magn Reson Med. 2010;64(6):1749-1759.

39. Shi X, Ma X, Wu W, Huang F, Yuan C, Guo H. Parallel imaging and compressed sensing combined framework for accelerating high-resolution diffusion tensor imaging using inter-image correlation. Magn Reson Med. 2015;73(5):1775-1785.

40. Ilicak E, Senel LK, Biyik E, Cukur T. Profile-encoding reconstruction for multiple-acquisition balanced steady-state free precession imaging. Magn Reson Med. 2017;78(4):1316-1329.

41. Liang D, Liu B, Wang J, Ying L. Accelerating SENSE using compressed sensing. Magn Reson Med. 2009;62(6):1574-1584.

42. Gamper U, Boesiger P, Kozerke S. Compressed sensing in dynamic MRI. Magn Reson Med. 2008;59(2):365-373.

43. Cukur T. Accelerated phase-cycled SSFP imaging with compressed sensing. IEEE Trans Med Imaging. 2015;34(1):107-115.

44. Cukur T, Lustig M, Saritas EU, Nishimura DG. Signal compensation and compressed sensing for magnetization-prepared MR angiography. IEEE Trans Med Imaging. 2011;30(5):1017-1027.

45. Block KT, Uecker M, Frahm J. Undersampled radial MRI with multiple coils. Iterative image reconstruction using a total variation constraint. Magn Reson Med. 2007;57(6):1086-1098.

46. Setsompop K, Kimmlingen R, Eberlein E, et al. Pushing the limits of in vivo diffusion MRI for the human Connectome project. Neuroimage. 2013;80: 220-233.

47. Chen SS, Donoho DL, Saunders MA. Atomic decomposition by basis pursuit. SIAM Journal on Scientific Computing. 1998;20(1):33-61.

48. Rudin LI, Osher S, Fatemi E. Nonlinear total variation based noise removal algorithms. Physica D: Nonlinear Phenomena. 1992;60(1):259-268.

49. Gong E, Huang F, Ying K, Wu W, Wang S, Yuan C. PROMISE: parallel-imaging and compressed-sensing reconstruction of multicontrast imaging using SharabIE information. Magn Reson Med. 2015;73(2):523-535.

50. Majumdar A, Ward RK. Compressed sensing of color images. Signal Processing. 2010;90(12):3122-3127.

51. Blomgren P, Chan TF. Color TV: total variation methods for restoration of vector-valued images. IEEE Trans Image Process. 1998;7(3):304-309.

52. Lai Z, Zhang X, Guo D, et al. Joint sparse reconstruction of multi-contrast MRI images with graph based redundant wavelet transform. BMC Med Imaging. 2018;18(1):7-7.

53. Lai Z, Qu X, Lu H, et al. Sparse MRI reconstruction using multi-contrast image guided graph representation. Magn Reson Imaging. 2017;43:95-104.

54. Ehrhardt MJ, Thielemans K, Pizarro L, et al. Joint reconstruction of PET-MRI by exploiting structural similarity. Inverse Problems. $2014 ; 31(1): 015001$.

55. Ehrhardt MJ, Betcke MM. Multicontrast MRI reconstruction with structure-guided total variation. SIAM Journal on Imaging Sciences. 2016;9(3): 1084-1106.

56. Mehranian A, Belzunce MA, Prieto C, Hammers A, Reader AJ. Synergistic PET and SENSE MR image reconstruction using joint Sparsity regularization. IEEE Trans Med Imaging. 2018;37(1):20-34.

57. Bredies K, Kunisch K, Pock T. Total generalized variation. SIAM Journal on Imaging Sciences. 2010;3(3):492-526. 
58. Li R, Li Y, Fang R, Zhang S, Pan H, Huang J. Fast Preconditioning for Accelerated Multi-contrast MRI Reconstruction. Paper presented at: Medical Image Computing and Computer-Assisted Intervention -- MICCAI 2015; 2015//, 2015; Cham.

59. Guven HE, Gungor A, Cetin M. An augmented Lagrangian method for complex-valued compressed SAR imaging. leee Transactions on Computational Imaging. 2016;2(3):235-250.

60. Uecker M, Ong F, Tamir J, et al. Berkeley advanced reconstruction toolbox. Paper presented at: proceedings of the 23rd annual meeting of ISMRM2015; Toronto. CANA.

61. Chatnuntawech I, Martin A, Bilgic B, Setsompop K, Adalsteinsson E, Schiavi E. Vectorial total generalized variation for accelerated multi-channel multicontrast MRI. Magn Reson Imaging. 2016;34(8):1161-1170.

62. Güngör A, Kopanoğlu E, Çukur T, Güven HE. Fast recovery of compressed multi-contrast magnetic resonance images. Paper presented at: SPIE Medical Imaging2017.

63. Uecker M, Lai P, Murphy MJ, et al. ESPIRiT--an eigenvalue approach to autocalibrating parallel MRI: where SENSE meets GRAPPA. Magn Reson Med. 2014;71(3):990-1001.

64. Kopanoglu E, Gungor A, Kilic T, et al. Multi-channel multi-contrast reconstructions via simultaneous use of individual and joint regularization terms. Paper presented at: proceedings of the 27th annual meeting of ISMRM2019; Montreal, Canada.

65. Kopanoglu E, Gungor A, Kilic T, Saritas EU, Cukur T, Guven HE. Joint reconstruction of multi-contrast images: compressive sensing reconstruction using both joint and individual regularization functions. Paper presented at: proceedings of the 25th annual meeting of ISMRM2017; Honolulu, HI, USA.

66. Boyd S. Distributed Optimization and Statistical Learning via the Alternating Direction Method of Multipliers. Foundations and Trends ${ }^{\circledR}$ in Machine Learning. 2010;3(1):1-122.

67. Xu Z, Figueiredo MA, Yuan X, Studer C, Goldstein T. Adaptive relaxed ADMM: Convergence theory and practical implementation. Paper presented at: 2017 IEEE Conference on Computer Vision and Pattern Recognition (CVPR)2017.

68. Aubert-Broche B, Griffin M, Pike GB, Evans AC, Collins DL. Twenty new digital brain phantoms for creation of validation image data bases. IEEE Trans Med Imaging. 2006;25(11):1410-1416.

69. Wang Z, Bovik AC, Sheikh HR, Simoncelli EP. Image quality assessment: from error visibility to structural similarity. IEEE Trans Image Process. 2004; 13(4):600-612.

70. Senel LK, Kilic T, Gungor A, et al. Statistically segregated k-space sampling for accelerating multiple-acquisition MRI. IEEE Trans Med Imaging. 2019; 38(7):1701-1714.

71. Aharon M, Elad M, Bruckstein A. K-SVD: an algorithm for designing overcomplete dictionaries for sparse representation. IEEE Transactions on Signal Processing. 2006;54(11):4311.

\section{SUPPORTING INFORMATION}

Additional supporting information may be found online in the Supporting Information section at the end of this article.

How to cite this article: Kopanoglu E, Güngör A, Kilic T, et al. Simultaneous use of individual and joint regularization terms in compressive sensing: Joint reconstruction of multi-channel multi-contrast MRI acquisitions. NMR in Biomedicine. 2020;33:e4247. https://doi.org/10.1002/ $\underline{\mathrm{nbm} .4247}$ 